\title{
Organization and Dynamics of The Spovaea Protein and its Surrounding Inner Membrane Lipids, Upon Germination of Bacillus Subtilis Spores
}

Juan Wen

University of Amsterdam

Norbert O.E. Vischer

University of Amsterdam

Arend L. Vos

University of Amsterdam

Erik. M. M. Manders

Confocal.nl

Peter Setlow

UConn Health

Stanley Brul ( $\square$ s.brul@uva.nl)

University of Amsterdam

Research Article

Keywords:

Posted Date: February 9th, 2022

DOI: https://doi.org/10.21203/rs.3.rs-1318522/v1

License: (1) This work is licensed under a Creative Commons Attribution 4.0 International License.

Read Full License 


\section{Abstract}

The SpoVA proteins make up a channel in the inner membrane (IM) of Bacillus subtilis spores. This channel responds to signals from activated germinant receptors (GRs), and allows release of $\mathrm{Ca}^{2+}$-DPA from the spore core during germination. In the current work, we studied the location and dynamics of SpoVAEa in dormant spores. Notably, the SpoVAEa-SGFP2 proteins were present in a single spot in spores, similar to the IM complex formed by all GRs termed the germinosome. However, while the GRs' spot remains in one location, the SpoVAEa-SGFP2 spot in the IM moved randomly with high frequency. It seems possible that this movement may be a means of communicating germination signals from the germinosome to the IM SpoVA channel, thus stimulating CaDPA release in germination. The dynamics of the SpoVAEa-SGFP2 and its surrounding IM region as stained by fluorescent dyes were also tracked during spore germination, as the dormant spore IM appeared to have an immobile germination related functional microdomain. This microdomain disappeared around the time of appearance of a germinated spore, and the loss of fluorescence of the IM with fluorescent dyes, as well as the appearance of peak SpoVAEa-SGFP2 fluorescent intensity occurred in parallel. These observed events were highly related to spores' rapid phase darkening, which is considered as due to rapid $\mathrm{Ca}^{2+} \mathrm{DPA}$ release. We also tested the response of SpoVAEa and the IM to thermal treatments at $40-80^{\circ} \mathrm{C}$. Heat treatment triggered an increase of green autofluorescence, which is speculated to be due to coat protein denaturation, and $80^{\circ} \mathrm{C}$ treatments induce the appearance of phase-grey-like spores. These spores presumably have a similar intracellular physical state as the phase grey spores detected in the germination but lack the functional proteins for further germination events.

\section{Introduction}

Bacillus subtilis, the model Gram-positive bacterium, has multiple complex responses to environmental stress and nutrient depletion. Notably, it can generate different subsets of cells, such as persisters, spores, and biofilms to promote survival in harsh environmental conditions [1]. Spores in particular are capable of maintaining metabolic dormancy for very long times by protecting their chromosomal DNA through its location in the low water environment of the spore core and its surrounding by multiple protective macromolecular layers [2]. From the outside in, these spore layers are the coat, outer membrane (OM), peptidoglycan (PG) cortex, germ cell wall, inner membrane (IM) and core [2]. Despite their potential long period of dormancy, these spores can be revived or germinated by many environmental cues, primarily small molecules termed germinants that signal the presence of a growth friendly environment, and they then resume cell growth [2].

The SpoVA channel is located in the IM, and has a crucial role in spore germination [3-7]. This channel functions to release the large pool of the 1:1 complex of $\mathrm{Ca}^{2+}$ and dipicolinic acid ( $\left.\mathrm{Ca}^{2+}-\mathrm{DPA}\right)$ from the spore core during germination $[5,8]$. Upon release of the $\mathrm{Ca}^{2+}-\mathrm{DPA}$, water is taken up and hydrolysis of the PG cortex begins. When the latter is complete the core water content has returned to the levels observed in vegetative cells $[2,8]$. Multiple signals can trigger the opening of the SpoVA channel, including 
activated germinant receptors (GRs), hydrolysis of the cortex PG and high hydrostatic pressure [2]. The SpoVA channel has seven subunits, A, B, C, D, Eb, Ea and F [6, 9]. SpoVAA, -B, -C, -Eb and -F are transmembrane IM proteins, one of which, SpoVAC, is a pressure-sensitive membrane channel protein [6, $7,10,11]$. SpoVAD and -Ea are hydrophilic proteins, located on the outer surface of the IM. Somehow there must be signal transduction from activated GRs to the SpoVA channel, but while there is some evidence for physical interaction between GRs and some SpoVA proteins [6, 12], how signal transduction takes place is not clear. $B$. subtilis spores have an estimated level of $\sim 7000$ molecules of SpoVA proteins, which is 7-10 times higher than the level of GRs [13]. Notably, all GRs in spores are in a complex in the IM that is generally present in only one or two immobile spots/spore termed germinosomes [14-17]. In contrast, by averaging hundreds of consecutive images, previous work indicated that SpoVAEa seems uniformly distributed throughout the IM [17]. In the current work, we created a SpoVAEa-SGFP2 reporter protein. The fusion protein was expressed in $B$. subtilis from the native spoVAEa locus and super resolution rescan confocal microscopy (RCM) was used to analyze SpoVAEa-SGFP2 location in spores.

As mentioned above, GRs are present in foci in the dormant spore IM, and our previous work found that $B$. subtilis spores' GerKB-SGFP2 foci reached peak fluorescence intensity around the 'time to germination', followed by the dispersion of the spots in a short time window in germinated spores [18]. The 'time to germination' was defined as the time needed for the spore to complete half of its rapid decline in phase brightness. The rapid decline of phase brightness is due to the refractility change of spores induced by $\mathrm{Ca}^{2+}$ DPA release and water uptake, and results in the change of a phase bright spore (dormant spore) into a phase dark spore (germinated spore) upon examination under a phase contrast microscope. The molecular basis of GR functioning in spore germination is still not resolved. However, we speculated that the increased GerKB-SGFP2 fluorescence is due to a relatively 'dramatic' environmental change in the physical state of the IM near the fusion protein [18]. Because both GRs and SpoVA proteins are IM proteins, we were curious to assess the dynamic response of SpoVAEa-SGFP2 fluorescence to the environmental changes occurring during germination. Time-lapse imaging was used to track both the refractility change of spores, and the dynamics of SpoVAEa-SGFP2 fluorescence during GR-triggered germination using phase contrast and widefield microscopy. The IM is undergoing dramatic

modifications during spore germination, including a 1.3 fold increase in its encompassed volume and the restoration of its full lipid mobility $[2,19]$. Consequently, we also tracked changes in the IM during germination. In addition, because previous work [20] showed that $40-80^{\circ} \mathrm{C}$ heat treatments of $B$. subtilis dormant spores resulted in spore heat activation of germination at temperatures $\leq 65^{\circ} \mathrm{C}$, caused a combination of heat activation and heat damage at $70-75^{\circ} \mathrm{C}$, and led to full heat inactivation at $80^{\circ} \mathrm{C}$, we also studied thermal effects on SpoVAEa-SGFP2 and membrane dye related fluorescence.

\section{Results}

\section{Location and the dynamics of SpoVAEa protein in dormant spores}


By using widefield microscopy, we found that the spore surface area did not have a uniform level of SpoVAEa-SGFP2. Next, we employed RCM with a scanning time of 2 sec per frame to obtain more detailed structural information [21]. Surprisingly, clusters of GFP fluorescence were observed, which clearly stood out from the background in dormant PS832 SpoVAEa-SGFP2 spores (Fig. 1A, left panel). By enlarging one of the spores, two spots were observed in the spore labelled 'a' (Fig. 1A, right panel). The full width at half maximum (FWHM) of the pronounced spot of spore 'a' was $220 \mathrm{~nm}$ (Fig. 1C). Due to the fact that the visualization of germination proteins can be interfered with by heavy autofluorescence from the spore coat, we also imaged SpoVAEa-SGFP2 in spores with a severely defective coat [22]. Similar fluorescent spot structures were found in the coat defective PS4150 SpoVAEa-SGFP2 spores (Fig. 1B, C). These spots were, however, not more pronounced indicating that our observations in wild-type spores were not majorly obscured by coat layers.

Previously, we showed that GerKB-SGFP2 proteins, one class of GRs, also presented themselves as 1-3 spots per spore [18]. These spots 'oscillated' at high frequency, but were confined only to a small area of the IM [18]. Here, the crucial question is whether SpoVAEa-SGFP2 is also at least somewhat mobile in the IM. To address this query, RCM microscopy was applied for high frequency imaging with a scanning time of 22.2 milliseconds per frame. Even more surprisingly, a single spot, traveling around the IM, i.e. not confined to a small area, was observed in the PS832 SpoVAEa-SGFP2 dormant spores when the spore was tracked for 1000 frames. We presume that we are the first to see this movement, as we imaged at very high time resolution. The SpoVAEa-SGFP2 fluorescent spot in the spore 'randomly' appeared in different time frames and different locations of the spore. Fig. 1D shows eight frames with a pronounced fluorescent spot, and the location differs in each frame. The measured FWHM of the spot in different frames varied from $96 \mathrm{~nm}$ to $263 \mathrm{~nm}$. Hence, the observed high frequency movement with longer exposure time, could explain why not all the spores in Fig. 1A and Fig. 1B, showed a spot structure, as well as the uniform distribution of SpoVAE, most likely SpoVAEa, in previous work [17]. It is, however, not clear how SpoVAEa is able to move at such a high frequency in an otherwise 'rigid' inner membrane [19, 23], although SpoVAEa appears to be on the outer surface of the IM [6]. Moreover, we cannot exclude that there are some 'free' SpoVAEa proteins distributed over the IM outside of the spot.

\section{Dynamics of SpoVAEa in the IM of $B$. subtilis spores during GR-triggered spore germination}

Previously, we found that during spore germination GerKB-SGFP2 spots gradually increased in fluorescence intensity [18]. This phenomenon is potentially related to the change of the spore's physical state in general and the IM in particular upon germination, because no new protein synthesis was observed [18]. It could be linked, for instance, to the increase in core water content and core $\mathrm{pH}$ due to the release of $\mathrm{Ca}^{2+}$-DPA and cortex hydrolysis. Here we used widefield microscopy to track the overall mean intensity of SpoVAEa-SGFP2 in spores during germination via GerB and GerK GRs by supplying the AGFK nutrient germinant cocktail (L-asparagine, glucose, fructose, and potassium chloride).

The phase brightness and SpoVAEa-SGFP2 fluorescence history of a single spore is shown in the timelapse image montage in Fig. 2A and 2C. By analyzing the brightness and fluorescence profiles of this 
spore, we observed that the peak fluorescence intensity of SpoVAEa-SGFP2 was reached before the appearance of the phase dark spore, followed by a slow decline of fluorescence intensity in the phase dark spore (Fig. 2A-D). The initiation of SpoVAEa-SGFP2 fluorescence increase was at the same time as the start of the spore's brightness rapid decline, which is considered as the start of rapid $\mathrm{Ca}^{2+}$-DPA release (Fig. 2B, D, E). In order to confirm the observed dynamics in the population, we synchronized SpoVAEaSGFPs fluorescence profiles by defining the $t=0$ as the 'time to germination', which is the time needed for the spore to complete half of its rapid decline in phase brightness (Fig. 2E). In the averaged trace of 418 germinating spores, the SpoVAEa-SGFP2 intensity increased sharply and the peak was around the 'time to germination' (Fig. 2F), while no SpoVAEa-SGFP2 synthesis was detected by western blot analysis (Fig. S1). Two independent batches of spores were analysed in this experiment. Thus, the increase of SpoVAEa-SGFP2 fluorescence intensity during germination is correlated with the rapid release of $\mathrm{Ca}^{2+}$ DPA. Western blot analysis in the current work, as well as previous work, did not detect a significant decrease of SpoVAEa level in germinated spores compared to dormant spores (Fig. S1), however, a decrease of SpoVAEa-SGFP2 fluorescence was observed in germinated spores (Fig. 2D, 2F) [6]. Subsequently, we found that photobleaching had a role in the observed fluorescence decrease (Fig. S2, S3), and photochemical alteration of both spore coat and SGFP2 potentially contributed to the photobleaching. A spore coat defective strain with no autofluorescence will likely be necessary to study the dynamics of SpoVAEa-SGFP2 in germinated spores [22, 24].

\section{Changes in IM structure upon triggering spore germination via GRs}

It is known that lipids in the $B$. subtilis dormant spore IM are largely immobile [2, 19]. Still, this IM is capable of increasing its surface area $~ 1.3$ fold upon the spore swelling due to spore core water uptake and cortex hydrolysis [2]. As indicated above, SpoVAEa-SGFP2 fluorescence reached peak intensity around 'time to germination'. Consequently, we decided to probe for a putative correlation between the SpoVAEa-SGFP2 peak fluorescence intensity and the change in mobility of the IM lipids. To that end, the IM of PS832 spores was stained with either the carbocyanine dye DilC 12 or the styryl dye FM5-95, which were added to a sporulating culture and hence incorporated into the IM upon the formation of the forespore, as has been shown in previous studies $[16,19]$. Any lipid probe present in the OM could be removed by extensive washing during spore purification [19].

We again used widefield microscopy to track the change in IM staining during germination. The fluorescence intensity of DilC $_{12}$ and FM5-95 stained spores dropped dramatically upon the start of the rapid decline in spore brightness, and the drop was completed around the 'time to germination' (Fig. 3, 4). In a word, just as with the dynamics of SpoVAEa-SGFP2, the change of the IM during germination is also highly correlated with the rapid $\mathrm{Ca}^{2+}{ }^{2}$ DPA release and cortex hydrolysis, which lead to the increase of spore core $\mathrm{pH}$, and water content [26, 27]. The FM5-95 stained spore also had a fluorescent spot, whereas DilC $_{12}$ spores had relative uniform staining (Fig. 3C, 4C). Regarding the fact that the FM5-95 dye was almost invisible in the germinated spore (Fig. 4C), we note that the hydrophobicity and affinity of FM5-95 for the IM lipids seems lower than that of DilC12. This implies also that FM5-95 prefers to bind in lipid 
domains with higher fluidity. Hence, the FM5-95 spot area could well be a fluid membrane domain, instead of the compressed IM. The disappearance of the FM5-95 spot in the phase dark spore, suggests that this region might be a germination related functional membrane microdomain (Fig. 4C).

\section{Response of SpoVAEa and probe stained IM to thermal treatments}

Our previous work showed that heating $B$. subtilis spores at $40-65^{\circ} \mathrm{C}$ promotes spore germination in a positive time/temperature dependent manner, higher heat temperatures resulted in both heat activation and heat damage, and eventually led to heat inactivation at $80^{\circ} \mathrm{C}$ [20]. The germination proteins and their surrounding IM are potential targets of the heat treatments. Here we studied whether heat treatments change the states of the spore's, SpoVAEa and the IM after 5 hours of heat treatment at $40-80^{\circ} \mathrm{C}$.

Significant drops of spore's absorbance at the population (Fig. 5A, 7A, 7E) and refractive index at single spore level (Fig. 5C, 5E, 7C, 7G) were detected in spores treated at $80^{\circ} \mathrm{C}$, and in some cases also in $75^{\circ} \mathrm{C}$ treated spores. Spores treated at $40-70^{\circ} \mathrm{C}$ morphologically looked similar upon the microscopical examination. Hence, we only present images of 65,75 , and $80^{\circ} \mathrm{C}$ heated spores as representative images (Fig. 6, 8). As shown in the phase contrast images (Fig. 6, 8), a subpopulation of phase-grey-like spores appeared in $80^{\circ} \mathrm{C}$ treated groups. These phase-grey-like spores are potentially spores that have lost $\mathrm{Ca}^{2+}-$ DPA, as reported previously $[28,29]$.

Heat treatments at $40-80^{\circ} \mathrm{C}$ led to a decrease of fluorescence intensity of coat defective PS4150 SpoVAEa-SGFP2 spores (Fig. 5F, 6D). However, we cannot exclude the possibility that the decreased SpoVAEa-SGFP2 spore fluorescence was caused by heat denaturation of SGFP2 [30]. This denaturation seems reversible at temperatures $\leq 65^{\circ} \mathrm{C}$, due to the fact that $65^{\circ} \mathrm{C}$ treated PS832 SpoVAEa-SGFP2 spores still exhibited similar fluorescence profiles as untreated spores during spore germination (Fig. S2, 2). However, it is not clear how spores maintain functionally active channel proteins during the heat activation process. Clearly, a pH and thermal stable fluorescent reporter will be important for future spore related research [31-33]. Notably, we tested the response of SpoVAEa-SGFP2 to heat in coat defective spores, because the change of SpoVAEa-SGFP2 fluorescence was masked by the enhanced green autofluorescence of the spore coat (Fig. 5,6). The increase of autofluorescence had a positive correlation with the heat treatment temperature. We speculate the enhanced autofluorescence is likely related to coat protein denaturation, since studies showed that a lethal thermal treatment induced significant protein denaturation in $B$. subtilis, $B$. cereus, and $B$. megaterium spores [28, 29]. Besides, the observed increased autofluorescence in PS4510 spores treated at $80^{\circ} \mathrm{C}$ is considered due to the response of either the residual outer coat or the inner coat layers to heat (Fig. 6C).

Compared to the untreated (ut) DilC $_{12}$ labeled spores, spores heated at $40-80^{\circ} \mathrm{C}$ exhibited slightly decreased fluorescence intensity (Fig. 7B, 7D, 8A). However, no correlation was detected between the drop of the phase contrast image brightness and changes in $\mathrm{DilC}_{12}$ fluorescence (Table.S2). In contrast, FM595 stained spores showed a continuous fluorescence decrease upon treatment at 70,75, and $80^{\circ} \mathrm{C}$ (Fig. 7F, 7H, 8B). The FM5-95 fluorescence had a positive correlation with the spore brightness of 70 and 
$75^{\circ} \mathrm{C}$ heated spores (Table.S2), Pearson correlation coefficients of 0.54 and 0.76 , respectively, and it was minimal in spores heated at $80^{\circ} \mathrm{C}$. As mentioned above, we speculate that spores with decreased brightness are potentially spores that have lost $\mathrm{Ca}^{2+}-\mathrm{DPA}$. Hence, the decrease of FM5-95 intensity presumably correlated with the release of $\mathrm{Ca}^{2+}-\mathrm{DPA}$, and the subsequent physical state changes in spores.

\section{Discussion}

Our work shows that at least one SpoVA subunit SpoVAEa, clustered in the IM as a spot undergoing high frequency movement in dormant $B$. subtilis spores. A germination related microdomain with higher fluidity was also observed in the spore IM. The rapid phase darkening of IM labeled spores, caused by the rapid release of $\mathrm{Ca}^{2+}$ DPA and by cortex hydrolysis, is accompanied by the loss of fluorescence in the IM, the disappearance of the IM fluid microdomain, and an increase in SpoVAEa-SGFP2 fluorescence intensity. Heat treatment at $65-80^{\circ} \mathrm{C}$ resulted in a temperature dependent increase in green autofluorescence, potentially caused by the degradation of coat proteins. Dormant spores heat treated at $80^{\circ} \mathrm{C}$ have a sub-population of phase-grey-like spores.

As mentioned above, a FM5-95 stained microdomain was observed in dormant spores, and this domain disappeared upon the appearance of the phase dark germinated spore. In addition, the microdomain likely has higher fluidity compared to the rest of the IM, and remains in a confined location in the IM before dispersing upon germination. This immobile behavior is likely in line with the 'germinosome' spot, in which GRs and their scaffold protein GerD are clustered together and are immobile [17]. In addition, our previous work showed that following spore germination the 'germinosome' agglomerates and diffuses in the same location of the IM, and gradually disperses $[15,18]$. Hence, there is a potential link between the germinosome and the fluid microdomain. To better understand this germination related membrane domain, efforts can take aim at answering the following questions. 1) Is the 'germinosome' spot truly located in the microdomain? 2) Does GerD have a function in recruiting the specific disordered lipids into this microdomain, as it does play such a role in maintaining the 'germinosome' spot? 3) When and how does the fluid microdomain form during sporulation?

Except for the microdomain in the IM, the spore IM itself still remains mysterious. Our current work, as well as previous publications showed that there is an expansion of the IM surrounded area and a reduced staining by lipid dyes in the IM during germination [19]. Notably, it has long been known that this dramatic increase of the IM surrounded area takes place without new lipid synthesis, and a recent electron microscopy study revealed an intracellular membrane structure in dormant spores below the IM $[19,34]$. This membrane structure, which contains at least one SpoVA protein, disappears upon spore core hydration, most likely due to integration with the IM [34]. The integration of the IM lipids during germination might have a role in the decline of the dye staining in the IM. A technique with better temporal and spatial resolution might reveal the specifics of the integration. Current work showed that SpoVAEa clusters in the IM as a spot, and is capable of moving in the 'gel state' IM randomly with high frequency. This random movement of the SpoVAEa spot might facilitate a physical interaction with both 
the immobile GR spots as well as the entire SpoVA protein channel, and thus communicate signals from the germinosome to the SpoVA CaDPA release channel. However, it is not clear how the SpoVA channel proteins interact with GRs, nor whether this interaction requires the integration of all SpoVA proteins either in the spot or dissociated in the IM, or even from the intracellular membrane structure. In addition, SpoVA channel proteins, synthesized in the developing forespores, are also crucial for import of $\mathrm{Ca}^{2+}$-DPA during sporulation [6, 35]. Revealing the mechanism of SpoVA spot formation in the forespore will contribute to the knowledge of both $\mathrm{Ca}^{2+}$-DPA uptake and release processes, as well as perhaps to an understanding of signal transduction between the germinosome and SpoVA channels.

We also made efforts to check the response of SpoVAEa-SGFP2 and the dye stained IM to heat treatments, which induced either heat activation or heat inactivation to spores. The thermal inactivation resulted in the appearance of phase-grey-like spores. However, no clear changes were observed in spores treated at heat activation temperatures $\left(40-65^{\circ} \mathrm{C}\right)$. Interestingly, the green autofluorescence, related to the proteinaceous coat, increased with the elevated heat temperatures. We speculate this phenomenon likely relates to coat protein' denaturation. Studying spore proteins' thermal stability profile on the proteome scale might provide a way to understand heat activation and inactivation at the molecular level.

\section{Materials And Methods}

\section{Bacterial strains, and culture conditions}

B. subtilis PS832 is a prototrophic 168 laboratory strain. Strain PS4150 is derived from PS832, carries cotE and gerE deletion mutations and is a coat defective mutant [22]. Strains PS832 SpoVAEa-SGFP2 and PS4150 SpoVAEa-SGFP2 were derived from PS832 and PS4150, respectively. These two strains contain a SpoVAEa-C-terminal SGFP2 fusion, integrated at the spoVAEa locus, and expressed under the control of the spoVA operon's promoter [36]. Spores of all strains were prepared in $2 \times S G$ sporulation medium at $37^{\circ} \mathrm{C}$ as described previously [37]. If required, the fluorescent dyes DilC $_{12}(1 \mu \mathrm{g} / \mathrm{ml}$, ThermoFisher) or FM5-95 ( $2 \mu \mathrm{g} / \mathrm{mL}$, ThermoFisher) were added to the sporulating culture, when it reached the peak optical density at $600 \mathrm{~nm}\left(\mathrm{OD}_{600}\right)$. Spores were harvested and purified, including centrifugation through HistodenZ as described previously [16]. Spores $\left(\mathrm{OD}_{600} \sim 60\right.$, in MIlliQ water) used in the current work had $\geq 98 \%$ dormant spores, and were essentially free of germinated spores, cells or cell debris as verified by phase contrast microscopy.

\section{Measuring the fluorescence of SpoVAEa-SGFP2 and the dye stained IM in heated spore populations}

Spores (OD600 $=\sim 1$, in MIlliQ water) were heated for $5 \mathrm{~h}$ at $40,50,60,65,70,75$ or $80^{\circ} \mathrm{C}$, followed by cooling in a water-ice bath $(\geqslant 15 \mathrm{~min})$. Spores $\left(\mathrm{OD}_{600}=\sim 1,150 \mu \mathrm{l} /\right.$ well) were added to a 96-well flatbottomed microtiter plate (black wall, Greiner Bio-One), and the optical density and fluorescence intensity were measured by a BioTek plate reader. Data were collected from at least two independent tests, each of them had three biological repeats. 


\section{Imaging And Image Analysis}

In current work, two microscopes were employed for imaging. The widefield microscope had a Nikon Ti Microscope, a NA1.45 plan Apo $\lambda$ 100× Oil Ph3 DM objective, and the rescan confocal microscope was equipped with a Nikon Ti Microscope and a NA1.49 SR Apo TIRF 100x objective. Spores were stabilized on a $1.5 \%$ agarose pad sealed in an air containing chamber as described previously [38]. If necessary, spores were heated for $5 \mathrm{~h}$ at $40,50,60,65,70,75$ and $80^{\circ} \mathrm{C}$, followed by cooling in a water-ice bath ( $\geqslant 15$ $\mathrm{min})$. In case of tracking spores' germination, HEPES buffer $(25 \mathrm{mM})$ supplemented with AGFK (Lasparagine, glucose, fructose, and potassium chloride, $10 \mathrm{mM}$ each) were provided in the agarose pad. The time lapse images were captured once every $1.5 \mathrm{~min}$ for $90 \mathrm{~min}$.

Time lapse images were analyzed by the ImageJ macro SporeTrackerB_06k [39]. In brief, spores were detected and marked in the first-time frame of the phase contrast images. For each spore, a phase contrast and a fluorescence montage stack were created to present the phase contrast brightness history, and the fluorescent dynamics of this spore (Fig. 2A, 2C). The phase contrast brightness profile and fluorescent profile of the spore were detected and stored by SporeTrackerB_06k for display and further analysis (Fig. 2B, 2D). Subsequently, the time of start $\left(T_{\text {RapidRelease }}\right)$ and $\left(T_{\text {PhaseDark }}\right)$ end of the rapid decline in the brightness profile were detected, and further the 'time to germination' was calculated for presenting the averaged fluorescence profile in the population (Fig. 2E) [24]. Here, the 'time to germination' $=1 / 2 \times\left(T_{\text {RapidRelease }}{ }^{+T_{\text {PhaseDark }}}\right)$. In order to present the fluorescent profile of the population, each spore's fluorescence profile was synchronized by defining $t=0$ min as the 'time to germination'. Eventually, averaged fluorescence traces vs time relative to germination were created to show the fluorescence profile in the population (Fig. 2F). Two channel images (phase contrast and fluorescent image) with single time frames were analyzed by ImageJ macro SporeAnalyzer. Spores were detected and marked in the phase contrast channel, followed by the detection of spore brightness and fluorescence intensity of each spore. Both macro runs in the background of ImageJ plugin ObjectJ.

\section{Western Blot Analysis}

B. subtilis PS832 SpoVAEa-SGFP2 spores were heat activated for $30 \mathrm{~min}$ at $70^{\circ} \mathrm{C}$, followed by cooling in a water-ice bath $(\geqslant 15 \mathrm{~min})$. Subsequently, spore germination $(\mathrm{OD} 600=\sim 30,100 \mu \mathrm{l})$ was triggered by Lasparagine, glucose, fructose, and potassium chloride, $10 \mathrm{mM}$ each (AGFK) in $5 \mathrm{ml}$ MOPS medium. Spores were collected after incubation at $37^{\circ} \mathrm{C}$ for $0,15,30$, and 60 min with continuous rotation at 200 $\mathrm{rpm}$. Spore lysates were obtained by the procedure of Troiano et al. [15]. Proteins from equal aliquots of the same amounts of spores were run on a Tricine-SDS-PAGE gel, and probed with rabbit polyclonal antiGFP antibody (Abcam) and HRP-conjugated goat anti-rabbit IgG H\&L (Abcam) on a PDVF membrane [40].

\section{Declarations}

\section{Author contributions}


J. Wen (data analysis and interpretation, manuscript writing), N.O.E. Vischer (image analysis), A.D. Vos (data analysis and interpretation), E.M.M. Manders (RCM microscopy), P. Setlow (data interpretation and manuscript editing) and S. Brul (data analysis, manuscript interpretation, editing and project supervision).

\section{Acknowledgements}

We thank the Van Leeuwenhoek Centre for Advanced Microscopy (LCAM) at the University of Amsterdam for the use of the widefield microscope, and the Confocal.nl for the use of the RCM microscope. We thank Ronald Breedijk from LCAM for the assistance for imaging. We thank Jeroen Kole from Confocal.nl for the RCM imaging. Juan Wen acknowledges the China Scholarship Council for a PhD fellowship.

\section{References}

1. Lopez, D., Vlamakis, H. \& Kolter R. Generation of multiple cell types in Bacillus subtilis. FEMS Microbiol. Rev. 33, 152-163 (2009).

2. Setlow, P., Wang, S. \& Li, Y-Q. Germination of spores of the orders Bacillales and Clostridiales. Annu. Rev. Microbiol.71, 459-477 (2017).

3. Zheng, L., Abhyankar, W., Ouwerling, N. Dekker, H. L., van Veen, H. et al. Bacillus subtilis spore inner membrane proteome. J. Proteome. Res. 15, 585-594 (2016).

4. Korza, G. \& Setlow, P. Topology and accessibility of germination proteins in the Bacillus subtilis spore inner membrane. J. Bacteriol.195, 1484-1491 (2013).

5. Francis, M.B. \& Sorg, J.A. Dipicolinic acid release by germinating spores occurs through a mechanosensing mechanism. mSphere. 1, e00306-16 (2016)

6. Perez-Valdespino, A., Li, Y., Setlow, B., Ghosh, S., Pan, D. et al. Function of the SpoVAEa and SpoVAF proteins of Bacillus subtilis spores. J. Bacteriol.196, 2077-2088 (2014)

7. Vepachedu, V.R. \& Setlow, P. Localization of SpoVAD to the inner membrane of spores of Bacillus subtilis. J. Bacteriol.87, 5677-5682 (2005).

8. Vepachedu, V.R. \& Setlow, P. Role of SpoVA proteins in release of dipicolinic acid during germination of Bacillus subtilis spores triggered by dodecylamine or lysozyme. J. Bacteriol.189, 1565-1572 (2007)

9. Fort, P. \& Errington J. Nucleotide sequence and complementation analysis of a polycistronic sporulation operon, spoVA, in Bacillus subtilis. J. Gen. Microbiol.131, 1091-1105 (1985).

10. Wang, G., Yi, X., Li, Y-Q \& Setlow, P. Germination of individual Bacillus subtilis spores with alterations in the GerD and SpoVA proteins, which are important in spore germination. J. Bacteriol. 193, 23012311 (2011).

11. Velásquez, J., Schuurman-Wolters, G., Birkner, J.P., Abee, T. \& Poolman, B. Bacillus subtilis spore protein SpoVAC functions as a mechanosensitive channel. Mol. Microbiol.92, 813-823 (2014). 
12. Vepachedu, V.R. \& Setlow, P. Analysis of interactions between nutrient germinant receptors and SpoVA proteins of Bacillus subtilis spores. FEMS Microbiol. Lett. 274, $42-47$ (2007).

13. Stewart, K.-A.V. \& Setlow, P. Numbers of individual nutrient germinant receptors and other germination proteins in spores of Bacillus subtilis. J. Bacteriol.195, 3575-3582 (2013).

14. Wang, Y., de Boer, R., Vischer, N., van Haastrecht, P., Setlow, P. \& et al. Visualization of germination proteins in putative Bacillus cereus germinosomes. Int. J. Mol. Sci.21, 5198 (2020).

15. Troiano, A.J. Jr., Zhang, J., Cowan, A.E., Yu, J. \& Setlow, P. Analysis of the dynamics of a Bacillus subtilis spore germination protein complex during spore germination and outgrowth. J. Bacteriol. 197, 252-261. (2015)

16. Wen, J., Pasman, R., Manders, E.M.M., Setlow, P. \& Brul, S. Visualization of germinosomes and the inner membrane in Bacillus subtilis spores. J. Vis. Exp. e59388. (2019).

17. Griffiths, K.K., Zhang, J., Cowan, A.E., Yu, J. \& Setlow, P. Germination proteins in the inner membrane of dormant Bacillus subtilis spores colocalize in a discrete cluster. Mol. Microbiol. 81, 1061-1077 (2011).

18. Breedijk, R.M.P., Wen, J., Krishnaswami, V., Bernas, T., Manders, E.M.M. et al. A live-cell superresolution technique demonstrated by imaging germinosomes in wild-type bacterial spores. Sci. Rep.10, 5312 (2020).

19. Cowan, A.E., Olivastro, E.M., Koppel, D.E., Loshon, C.A., Setlow. B. et al. Lipids in the inner membrane of dormant spores of Bacillus species are largely immobile. Proc. Natl. Acad. Sci. 101, 7733-7738 (2004).

20. Wen, J., Smelt, J.P.P.M., Vischer, N.O.E., de Vos, A.L. et al. Heat activation and inactivation of bacterial spores. Is there an overlap? Appl. Environ. Microbiol. (2022); in press.

21. De Luca, G.M.R., Breedijk R.M.P., Brandt, R.A.J., Zeelenberg, C.H.C., de Jong, B.E. et al. Re-scan confocal microscopy: scanning twice for better resolution. Biomed. Opt. Express. 4, 2644-2656 (2013).

22. Ghosh, S., Setlow, B., Wahome, P.G., Cowan, A.E., Plomp, M. et al. Characterization of spores of Bacillus subtilis that lack most coat layers. J. Bacteriol.190, 6741-6748 (2008)

23. Loison, P., Hosny, N.A., Gervais, P., Champion, D., Kuimova, M.K. et al. Direct investigation of viscosity of an atypical inner membrane of Bacillus spores: a molecular rotor/FLIM study. Biochim. Biophys. Acta.1828, 2436-2443 (2013).

24. Magge, A., Setlow, B., Cowan, A.E. \& Setlow P. Analysis of dye binding by and membrane potential in spores of Bacillus species. J. Appl. Microbiol.106, 814-824 (2009)

25. Wang, S., Setlow, P. \& Li, Y-Q. Slow leakage of Ca-dipicolinic acid from individual Bacillus spores during initiation of spore germination. J. Bacteriol. 197, 1095-1103 (2015).

26. van Beilen, J.W.A. \& Brul, S. Compartment-specific pH monitoring in Bacillus subtilis using fluorescent sensor proteins: a tool to analyze the antibacterial effect of weak organic acids. Front. Microbiol.4, 157 (2013) 
27. Setlow, B. \& Setlow P. Measurements of the $\mathrm{pH}$ within dormant and germinated bacterial spores. Proc. Natl. Acad. Sci.77: 2474-2476 (1980)

28. Coleman W.H., Chen, D., Li, Y-Q, Cowan, A.E. \& Setlow, P. How moist heat kills spores of Bacillus subtilis. J. Bacteriol.189, 8458-8466 (2007).

29. Coleman, W.H., Zhang, P., Li, Y-Q. \& Setlow, P. Mechanism of killing of spores of Bacillus cereus and Bacillus megaterium by wet heat. Lett. Appl. Microbiol.50, 507-514 (2010).

30. Alkaabi, K.M., Yafea, A. \& Ashraf, S.S. Effect of pH on thermal- and chemical-induced denaturation of GFP. Appl. Biochem. Biotechnol.126, 149-156 (2005).

31. Cava, F., de Pedro, M.A., Blas-Galindo, E., Waldo, G.S., Westblade, L.F. et al. Expression and use of superfolder green fluorescent protein at high temperatures in vivo: a tool to study extreme thermophile biology. Environ. Microbiol.10, 605-613 (2008).

32. Frenzel, E., Legebeke, J., van Stralen, A., van Kranenburg, R. \& Kuipers, O.P. In vivo selection of sfGFP variants with improved and reliable functionality in industrially important thermophilic bacteria. Biotechnol. Biofuels.11, 1-19 (2018).

33. Doherty, G.P., Bailey, K. \& Lewis P.J. Stage-specific fluorescence intensity of GFP and mCherry during sporulation In Bacillus subtilis. BMC Res. Notes. 3,303. (2010).

34. Laue, M., Han, H.-M., Dittmann, C. \& Setlow, P. Intracellular membranes of bacterial endospores are reservoirs for spore core membrane expansion during spore germination. Sci. Rep. 8,11388 (2018).

35. Ramírez-Guadiana, F.H., Meeske, A.J., Rodrigues, C.D.A., Barajas-Ornelas, R.D.C., Kruse, A.C. \& et al. A two-step transport pathway allows the mother cell to nurture the developing spore in Bacillus subtilis. PLoS Genet.13, e1007015 (2017).

36. Kremers, G-J, Goedhart, J., van den Heuvel, D.J., Gerritsen, H.C. \& Gadella, T.W.J. Jr. Improved green and blue fluorescent proteins for expression in bacteria and mammalian cells. Biochemistry.46, 3775-3783 (2007).

37. Abhyankar, W.R., Kamphorst, K., Swarge, B.N., van Veen, H., van der Wel, N.N. et al. The influence of sporulation conditions on the spore coat protein composition of Bacillus subtilis spores. Front. Microbiol. 7,1636. (2016).

38. Pandey, R., Ter Beek, A., Vischer, N.O.E., Smelt, J.P.P.M., Brul, S. et al. Live cell imaging of germination and outgrowth of individual Bacillus subtilis spores; the effect of heat stress quantitatively analyzed with SporeTracker. PLoS One.8, e58972. (2013).

39. Omardien, S., Ter Beek, A., Vischer, N., Montijn, R., Schuren, F. et al. Evaluating novel synthetic compounds active against Bacillus subtilis and Bacillus cereus spores using Live imaging with SporeTrackerX. Sci. Rep.8, 9128. (2018).

40. Schägger, H. Tricine-SDS-PAGE. Nat. Protoc.1, 16-22 (2006).

\section{Figures}




\section{Figure 1}

Organization of SpoVAEa-SGFP2 in dormant spores in a region of increased fluorescence (a fluorescent 'spot'). (A) The RCM fluorescence image of PS832 SpoVAEa-SGFP2 spores. The spore in the inset ' $a$ ' is the enlarged view of the spore indicated by the grey arrow in the left panel. (B) The RCM fluorescence image of PS4150 SpoVAEa-SGFP2 spores. The spore in the inset ' $b$ ' is the enlarged view of the spore indicated by the grey arrow in the left panel. (C) The plot profiles of the pronounced SpoVAEa-SGFP2 spot of spores ' $a$ ' and ' $b$ '. (D) The RCM high frequency time-lapse images ( $22.2 \mathrm{~ms} / \mathrm{frame})$ of a PS832 SpoVAEa-SGFP2 dormant spore. The presented images represent frames in which the strong SpoVAEaSGFP2 spot appears during the high frequency time-lapse track. The relative FWHM of the SpoVAEaSGFP2 spot in each frame is indicated at the bottom of each image.

\section{Figure 2}

Dynamic behaviour of SpoVAEa-SGFP2 during spore germination. Spore germination was triggered by (10 mM each) AGFK without heat activation treatment. (A) Phase contrast time lapse images of a single PS832 SpoVAEa-SGFP2 spore. (B) The brightness profile corresponding to the images shown in panel A. (C) The fluorescence time lapse images of the same spore shown in panel A. (D) The SpoVAEa-SGFP2 fluorescence intensity profile corresponding to images in panel C. (E). Parameters detected by SporeTrackerX, and their corresponding positions in spore germination. As shown in the schematic, upon germination commitment, slow leakage of $\mathrm{Ca}^{2+} \mathrm{DPA}$ from the spore core begins, followed by the rapid release of the remaining $\mathrm{Ca}^{2+}{ }^{2+} \mathrm{PA}$ and then spore cortex hydrolysis [25]. The latter two events result in the rapid decline in the brightness of spores in phase contrast microscopy. Here, SporeTrackerB_06K detected

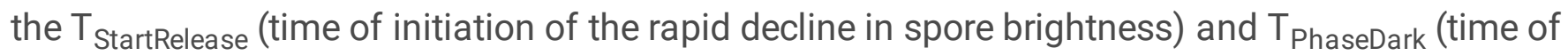
completion of spore brightness rapid decline) in the brightness profile, and further calculated the 'time to germination' for presenting data for the population. Here, the 'time to germination' is defined as the time needed for the spore to complete half of its rapid decline in phase brightness. The magenta arrow here and in (A) indicates the 'time to germination'. ( $F$ ) Average of 418 synchronized single SpoVAEa-SGFP2 spore fluorescence intensity traces. Synchronization defines $\mathrm{t}=0 \mathrm{~min}$ as the 'time to germination'. 613 spores were tracked by microscopy for 90 min and $68.2 \%$ of them completed germination.

\section{Figure 3}

Dynamic behavior of the DilC $_{12}$ stained wild-type spore IM during germination. Spore germination was triggered by (10 mM each) AGFK as described in Methods. (A) Phase brightness of a PS832 (DilC ${ }_{12}$ ) spore; the magenta triangle denotes the 'time to germination'. (B) The spore brightness profile corresponding to the images shown in panel A. (C) The fluorescence time lapse images of the spore 
shown in panel A. (D) The DilC 12 stained IM intensity profile corresponding to images in panel C; the magenta arrow indicates the 'time to germination'. (E) Average of 170 synchronized single DilC $_{12}$ spore fluorescence intensity traces. Synchronization defines $t=0 \mathrm{~min}$ as the 'time to germination'. 289 spores were tracked by microscopy for $90 \mathrm{~min}$, and $58.8 \%$ of them completed germination.

\section{Figure 4}

Dynamic behavior of the FM5-95 stained IM during wild-type spore germination. Spore germination was triggered by (10 mM each) AGFK as described in Methods. (A) Phase brightness of a PS832 (FM5-95) spore; the magenta arrow denotes the 'time to germination'. (B) The spore brightness profile corresponding to the images shown in panel A. (C) The fluorescence time lapse images of the same spore shown in panel A. (D) The FM5-95 stained IM intensity profile corresponding to images in the panel C; the magenta arrow indicates the 'time to germination'. (E) Average of 277 synchronized single FM5-95 spore fluorescence intensity traces. Synchronization defines $\mathrm{t}=0 \mathrm{~min}$ as the 'time to germination'. 369 spores were tracked by microscopy for $90 \mathrm{~min}$, and $75.1 \%$ of them completed germination.

\section{Figure 5}

Changes in SpoVAEa-SGFP2 dormant spore refractility and fluorescence intensity at population and single spore levels after 5 hours of heat treatment at various temperatures. (A) The change in OD at $600 \mathrm{~nm}$ of a PS832 SpoVAEa-SGFP2 dormant spore population measured by a plate reader. Untreated

PS832 spores were used as control. (B) The corresponding fluorescence intensity of spores in panel A. (C) The change of brightness of PS832 SpoVAEa-SGFP2 dormant spores at the single spore level measured by phase contrast microscopy. Here, PS832 spores with different heat treatments were employed as controls and $\geq 444$ spores were examined in each group. (D) The corresponding intensity of spores in panel C measured by widefield microscopy. (E) The brightness of PS4150 SpoVAEa-SGFP2 dormant spores at the single spore level. Here, PS4150 spores with different treatments were employed as control and $\geq 937$ spores were examined in each group. (F) The corresponding fluorescence intensity of spores in panel E. The number of spores detected by microscopy is presented in Table. S1.

\section{Figure 6}

The effect of 5 hours of heat-treatment on PS832 SpoVAEa-SGFP2 spores. The phase contrast (PC) and widefield fluorescence (Fluo) images of spores of wild type strain PS832 (A), PS832 SpoVAEa-SGFP2 (B), PS4150 (C), and PS4150 SpoVAEa-SGFP2 (D) after different heat treatments. (A) and (B) are the images 
corresponding to Fig. 5C-5D. (B) and (C) are the images corresponding to Fig. 5E-5F. The fluorescent image intensity display range for each strain is the same.

\section{Figure 7}

The change of lipid probe stained dormant spore refractility and fluorescence intensity at population and single spore levels after 5 hours of treatment at various temperatures. (A) The change in $\mathrm{OD}_{600}$ of PS832 $\left(\right.$ DilC $\left._{12}\right)$ dormant spore populations measured by a plate reader. Untreated PS832 spores were used as control. (B) The corresponding fluorescence intensity of spores in panel A. (C) The change of individual PS832 ( DilC $_{12}$ ) spores' brightness measured by a phase contrast microscopy. $\geq 612$ spores were examined in each group. (D) The corresponding fluorescence intensity of spores in panel C. (E) The brightness of individual PS832 (FM5-95) dormant spores. $\geq 932$ spores were examined in each group. (F) The corresponding fluorescence intensity of spores in panel $\mathrm{E}$. The numbers of individual spores examined by microscopy are given in Table. $\mathrm{S} 2$.

\section{Figure 8}

The effect of 5 hours of heat-treatment on the fluorescence intensity of IM probe stained spores. The phase contrast (PC) and widefield fluorescence (Fluo) images of wild type PS832 spores stained by DilC $_{12}(A)$, and FM5-95 (B) after different heat treatments: (A) images corresponding to Fig. 7C-7D; and (B) images corresponding to Fig. 7G-7H. The fluorescence images displayed for DilC ${ }_{12}$ spores are all in the same range, whereas, the FM5-95 fluorescence images displayed are in different ranges due to the big decline in brightness at higher temperatures.

\section{Supplementary Files}

This is a list of supplementary files associated with this preprint. Click to download.

- SPOVAEaBsubtilisBioRXivssupplementaryinformtionScientificReports.docx 